Conclusions We found some suggestion of a reduced risk of childhood asthma at age 7 with higher maternal plasma levels of omega- 3 and a higher ratio of omega- 3 to omega- 6 PUFAs.

\section{PS-062 WITHDRAWN}

\section{PS-063 VARIABILITY IN BREAST MILK COMPOSITION BASED ON MOTHER AND NEONATE CONDITIONS: A DESCRIPTIVE STUDY}

E Martín Alvarez, M Peña-Caballero, E Nievas-Cazorla, L Zamorano-Bonilla, L Serrano-López, JA Hurtado-Suazo. Paediatric, Hospital Universitario Virgen de Las Nieves, Granada, Spain

10.1136/archdischild-2014-307384.359

Background Recent studies on the field of evolutive biology have shown that the individual variability in breast milk (BM) composition may reflect mother or neonate characteristics. These results are focused on Trivers and Willard's evolutive theory (1973): natural choice may favour the inversion of the breastfeeding pattern.

Objective To prove that BM composition may be altered on the basis of neonate sex, mother age or number of children.

Study Design/methods Descriptive study of BM composition donated in a hospital breast milk bank from April 2013 to January 2014. Different variables were registered: Nutritional, mother (age) and neonate variables (sex and order of birth).

Results 280 BM donation sets performed with 79 mothers were analysed. From the overall of women, $46,8 \%$ were mothers who had daughters and 53,2\% had sons. It was their first child in 37 mothers. It could not be observed the correlation between mother age and nutritional values. In respect to the order of birth, it has been shown that the amount of fatty acids is much higher in first borns than in each child born subsequently (p0,027). Nevertheless, it has been shown that BM obtained from mothers who had daughters has a much higher protein $(\mathrm{p} 0,009)$, lactose content $(\mathrm{p} 0,055)$ and caloric value $(\mathrm{p} 0,05)$ than the one obtained from sons.

Conclusions BM composition is affected by sex and order of birth. The fatty acids concentration diminishes with the birth of each subsequent child. Therefore this research has shown nutritional differences in BM composition, favouring daughters.

\section{General Paediatric Surgery}

\section{PS-064 DIAGNOSTIC VALUES OF ALVARADO SCORING SYSTEM, ULTRASOUND AND C-REACTIVE PROTEIN IN PAEDIATRIC ACUTE APPENDICITIS}

A Zviedre, A Engelis, A Petersons. Pediatric Surgery, University Children's Hospital Riga Stradins University, Riga, Latvia

\subsection{6/archdischild-2014-307384.360}

Background and aim Ultrasound (US) and Alvarado scoring system (ASS) are very helpful in making the diagnosis of Acute appendicitis (AAp). Therefore important evaluate the usefulness of ASS, US and C-reactive protein (CRP) in AAp diagnostics for children (7-18 years).
Methods 46 patients with clinically suspected AAp were enrolled in the prospective study (January 2010-December 2013). Each patient underwent abdominal US and was evaluated for the ASS criteria and for CRP. The ASS is based on three symptoms, three signs, and two laboratory findings. Sensitivity and specifity of the diagnostic tests were assessed. The study was approved by the Institutional Ethics Review Board.

Results $58.7 \%$ of our patients were boys, $41.3 \%$ - girls, the mean age was 12.91 years (SD 3.2). 47.8\% had AAp and underwent appendectomy. The positive predictive value (PPV) for US was $95.5 \%$ and the negative predictive value (NPV) - 75\%. The sensitivity for diagnosing AAp by US - $77.82 \%$, the specificity 94.7\%. Anorexia and leukocytosis were significantly related to AAp ( $p<0.01)$. The sensitivity was $41.0 \%$, specificity $71.4 \%$, PPV 94.1\%, NPV $20.7 \%$ for the ASS with a cutoff point of 7 . Of patients with AAp and with ASS score $\geq 7$, two had a normal CRP, 12 patients with ASS scores $<7$ had CRP $\geq 5 \mathrm{mg} / \mathrm{L}$. The sensitivity was $75.0 \%$, specificity $83.3 \%$, PPV $75.0 \%$, NPV $33.3 \%$.

Conclusions ASS should be used in clinical practice for the patient's benefit. US provides reliable findings for helping to diagnose AAp if ASS score $\geq 7$ and CRP value is normal.

\section{PS-065 ULTRASONOGRAPHY AND PAEDIATRIC APPENDICITIS: A STORY OF GREAT EXPECTATIONS AND THE PHANTOM APPENDIX!}

${ }^{1} \mathrm{C}$ Beardsley, ${ }^{1} \mathrm{~A}$ Dillon, ${ }^{2} \mathrm{~F}$ Nguyen, ${ }^{3} \mathrm{GDH}$ Croaker. ${ }^{1}$ General Surgery/Paediatric Surgery, Canberra Hospital, Canberra, Australia; 'Paediatric Surgery, Canberra Hospital, Canberra, Australia; ${ }^{3}$ Paediatric Surgery, Canberra Hospital, Canberra, Australia

\subsection{6/archdischild-2014-307384.361}

Background and aims Appendicitis is the most common surgical emergency in children yet the diagnostic workup of children varies greatly among major centres internationally. Once heralded as a revolution in diagnosis, the results of its clinical application have been conflicting. Intra-operator variability has a significant effect on appendix visualisation, with some centres quoting visualisation rates as high as $80 \%$ and as others as low as $30 \%$. We had anecdotally observed that many ultrasounds ordered at our centre were not visualising the appendix and wanted to determine our visualisation rate and how it affects the utility of ultrasound in clinical practice.

Methods 10 year retrospective review of all children, presenting to the Canberra Hospital with right sided or periumbilical abdominal pain between 2002 and 2012, where ultrasonography was utilised in the confirmation or refutation of appendicitis as a diagnosis.

Results 737 ultrasound scans were reviewed. Rates of ultrasonography doubled over the period but appendix visualisation rate dropped from $42 \%$ to $25 \%$. Sensitivity is $96 \%$ when the appendix was visualised for all tests but only $71 \%$ when a negative result included non-visualised test. The incidence of appendicitis was $21 \%$ in the total cohort, and $6 \%$ in the nonvisualisation group.

Conclusion Ultrasonography is a theoretically appealing tool for paediatric appendicitis, if and only if, the appendix is visualised. If the incidence of appendicitis in the non-visualised group approaches that of the general population, a non-visualised test provides no reassurance whatsoever, and appendicitis remains a diagnostic phantom for the clinician. 